\title{
Herkunft und Zukunft der Sozialen Marktwirtschaft
}

\author{
Dominik Geppert • Hans Jörg Hennecke
}

Angenommen: 6. Mai 2021 / Online publiziert: 21. Mai 2021

(C) Der/die Autor(en) 2021

\section{1}

Die Corona-Pandemie hat die stabilitätsverwöhnte deutsche Wirtschafts- und Sozialpolitik im Frühjahr 2020 vor eine gänzlich unerwartete Herausforderung gestellt. Ihre unmittelbaren konjunkturellen und arbeitsmarktpolitischen Auswirkungen sind in der Wirtschaftsgeschichte der Bundesrepublik allenfalls mit den Erschütterungen vergleichbar, die nach 2008 durch die Finanzmarkt- und Staatsschuldenkrise ausgelöst wurden. Wie damals waren es nicht nur kurzfristige Auswirkungen wie das Hochschnellen der Kurzarbeit oder der Einbruch der Konjunkturindikatoren, an denen das Ausmaß der Krise abzumessen war. Es traten auch Fernwirkungen institutioneller und konzeptioneller Art ein, die die Wirtschafts- und Sozialordnung strukturell und dauerhaft verändern können.

Die Krise führte zu einer Neubestimmung der Rolle des Staates bei der Stabilisierung und Transformation der Wirtschaft, und sie tat dies nicht nur auf nationaler Ebene, sondern mittels neuer Vereinbarungen zur Geld- und Konjunkturpolitik auch auf europäischer Ebene. Längst zeichnet sich ab, dass sich die Entwicklungen, die nach 2008 im Zuge der kumulierenden „Rettungspolitik“ der europäischen Institutionen einsetzten, durch die konjunkturpolitischen Reaktionen auf die CoronaPandemie im Jahre 2020 verfestigt und verstärkt wurden. Ordnungsvorstellungen, die das Verhältnis von Staat, Wirtschaft und Gesellschaft justieren, befinden sich offenkundig im Umbruch - hin zu Konzeptionen, die eine aktivere Rolle des Staates zur Konjunkturstabilisierung und zur Steuerung von Transformations- und Innova-

Dominik Geppert $(\bowtie)$

Universität Potsdam, Potsdam, Deutschland

E-Mail: dgeppert@uni-potsdam.de

Hans Jörg Hennecke

Universität Rostock, Rostock, Deutschland

E-Mail: hans-joerg.hennecke@uni-rostock.de 
tionsprozessen vorsehen und weniger Scheu davor haben, die Staatstätigkeit auszudehnen, die Hürden für Staatsverschuldung abzusenken und daraus resultierende Kosten und Haftungsrisiken zu kollektivieren.

Hierbei treten verschärfte Konflikte zwischen „südeuropäischen“ und „,nordeuropäischen“ Positionen zur Geldpolitik, zur Vergemeinschaftung der Konjunkturpolitik und zur Staatsschuldenfinanzierung zutage, die erhebliche Sprengkraft für die europäische Integration entfalten können. Die europäische Integration dehnt sich offenkundig auf Bereiche aus, für die es keinen tragfähigen Konsens der Ordnungsvorstellungen mehr gibt. Aktuell werden diese Spannungslagen im Ringen um ,strategische Autonomie“ der Europäischen Union deutlich: Während auf der einen Seite die Offenheit der Märkte im Sinne des Freihandels für die Außenbeziehungen der EU betont wird, treten auf der anderen Seite Vorstellungen hervor, die in bestimmten industriellen Bereichen eine Abschottung nach außen und eine kleinteilige politische Steuerung im Inneren favorisieren.

Entgegen vieler Deutungen, die seit Schaffung der europäischen Währungsunion aus Frankreich, Italien oder Griechenland zu vernehmen waren, spielt Deutschland auf der europäischen Ebene schon seit einiger Zeit eine eher unentschiedene und kompromissbereite Rolle. Schon vor dem Ausbruch der Corona-Pandemie zeichnete sich eine Annäherung der wirtschafts- und integrationspolitischen Grundvorstellungen Deutschland an diejenigen Frankreichs ab. Im bilateralen „Vertrag von Aachen“ aus dem Jahre 2019 war diese Annäherung bereits ebenso angelegt wie in den Vorstellungen zur Industrie- und Innovationspolitik in der ,Nationalen Industriestrategie“ (BMWi 2019) aus demselben Jahr. Nach dem Vollzug des „Brexits“ hat das eher liberale Lager innerhalb der EU an Gewicht verloren. Für die EU dürfte dies auf lange Sicht kostspieliger und nachteiliger sein als der höhere Aufwand an Zollmodalitäten an der neuen EU-Außengrenze.

Am Ende der pragmatisch-passiven Ära Merkel stellt sich die Frage, ob all diese Entwicklungen eine angemessene Transformation und Modernisierung des Leitbildes der „Sozialen Marktwirtschaft“ darstellen oder ob es sich um einen grundlegenden Kurswechsel handelt, der das Land noch weiter als ohnehin schon von seinen konstitutiven Vorstellungen zur Wirtschafts- und Sozialordnung wegführt und vorhandene Strukturprobleme möglicherweise verschärft. Die politischen und wissenschaftlichen Diskussionen darüber werden auf die eine oder Weise die historische Bilanz der langen Ära Merkel prägen - sei es, dass sich das Bild einer pragmatisch-vorsichtigen Politik der Krisenreaktionen durchsetzt, bei denen das Land alles in allem passabel durch die ersten beiden Jahrzehnte des 21. Jahrhunderts gekommen ist, oder sei es, dass sich eher das Bild einer Ära der verpassten Chancen, der unterlassenen Reformen und der aufgestauten Probleme durchsetzen wird.

Die Einordnung der aktuellen Krisenpolitik und der aus ihr abgeleiteten neuen Strategien in die Traditionslinien der deutschen Wirtschafts- und Sozialpolitik fällt schon allein deshalb nicht leicht, weil der Begriff der "Sozialen Marktwirtschaft" ausgesprochen schillernd ist und mit ihm von je her eine wechselhafte Sprachge- 
brauchspolitik betrieben worden ist. Im politischen Raum wird der Begriff bisweilen lediglich als affirmative Legitimationsformel für den jeweiligen Ist-Zustand der Wirtschafts- und Sozialpolitik verwendet. Wo er hingegen als Kontrastfolie zum Status quo dient und Veränderungsforderungen rechtfertigen soll, kennzeichnet er mal den Anspruch auf freiheitlich ausgerichtete Reformen, mal aber auch den Anspruch auf eine sozialpolitische Einhegung der Marktwirtschaft.

Diese Unbestimmtheit gründet in dem politischen Erfolg der Sprachschöpfung. Alle Parteien nehmen heutzutage den Begriff für sich in Anspruch und versuchen, die Deutungshoheit über ihn zu erringen. Die „Soziale Marktwirtschaft“ ist damit nur ein besonders einleuchtendes Beispiel dafür, dass viele politische Begriffe nicht wirklich dazu geeignet sind, unterschiedliche Ordnungsvorstellungen eindeutig voneinander abzugrenzen, und dass ihr politischer Gebrauchswert oftmals gerade darin liegt, dass sie die Unterschiede zwischen konkurrierenden Ordnungsvorstellungen verwischen (Oakeshott 2000). Der klassische Liberale Friedrich August von Hayek sprach sogar despektierlich von dem Wieselwort „sozial“, das jeden Begriff - also auch die „Marktwirtschaft“ -, dem es vorangestellt werde, ausschlürfe und seines ursprünglichen Sinnes entleere (Hayek 1976). Und in der Tat: Je politisch attraktiver der Begriff „Soziale Marktwirtschaft“" wurde, desto unklarer wurde, was damit gemeint sei und was politische Kräfte, die ihn für sich in Anspruch nehmen, damit tatsächlich im Schilde führen.

Die integrative, befriedende Absicht der Begriffsschöpfung war schon bei Alfred Müller-Armack, der den Begriff nach dem Zweiten Weltkrieg zwar nicht erfand, aber prägte, angelegt, bevor Ludwig Erhard ihn sich zur Kennzeichnung seiner Politik zu eigen machte. Aber Erhards Politik war seit 1948 hochkontrovers und wurde von seinen Gegnern erbittert bekämpft. 1949, als die erste Bundestagswahl stattfand, wurde die „Soziale Marktwirtschaft“ plakativ der „Zwangswirtschaft“ gegenübergestellt und trug maßgeblich zu der starken, von Konrad Adenauer gewollten Polarisierung im Wahlkampf bei. Dadurch dass Erhards Politik von den bürgerlichen Parteien CDU, CSU, FDP und DP programmatisch aufgegriffen wurde, war einer Großen Koalition der Weg verbaut. Erst mit dem wachsenden und unbezweifelbaren Erfolg der praktischen Wirtschafts-, Finanz- und Sozialpolitik der frühen Bundesrepublik wandelte sich der Begriff zu einer politischen Integrationsformel, deren Deutungshoheit schließlich von allen Parteien beansprucht wurde. Ob Karl Schiller (1964), Bodo Hombach (1998), Sahra Wagenknecht (2011) oder neuerdings Robert Habeck (2019): Auch weit über das programmatische Spektrum von Union und FDP hinaus bezogen sich Wirtschaftspolitiker und programmatische Vordenker der unterschiedlichsten Parteien auf den Begriff der ,Sozialen Marktwirtschaft“ und machten ihn sich in wechselnden Interpretationen zu Eigen.

Aussagekräftiger als die Begriffsverwendung selbst war daher immer die Frage, in welcher Abgrenzung der Begriff jeweils verwendet wurde. Hier zeigten sich auch innerhalb der Programmdebatte der Union, die den Begriff besonders pflegt, immer wieder markante Unterschiede (vgl. exemplarisch z. B. Merz 2010 und Rüttgers 2007). Die Kontraststellung zu Begriffen wie ,Zwangswirtschaft“, „Planwirtschaft““ oder „Totalitarismus“ verwies immer darauf, dass unter „Sozialer Marktwirtschaft“ im Kern ein marktwirtschaftliches und freiheitliches Ordnungsmodell verstanden wurde und dass dieses Konzept eine Facette des „Neoliberalismus“ des 20. Jahr- 
hunderts war. Diese Deutung war freilich zu keinem Zeitpunkt unumstritten. Es gab immer auch eine Verwendungsweise des Begriffs, die auf eine Überwindung der Gegensätze zwischen liberalen und sozialistischen Vorstellungen abzielte, eine Synthese oder einen ,dritten Weg“ zu bezeichnen versuchte. Und es gab - insbesondere im Gefolge der Finanzmarktkrise von 2008 - Verwendungsweisen, die die ,Soziale Marktwirtschaft“ marktwirtschaftlich konnotierten Begriffen wie „Globalisierung“, „Kapitalismus“ oder „Neoliberalismus“ gegenüberstellten. Dies geschah nicht selten unter Bejahung eines ,rheinischen“, also eines frankophilen und merkantilistisch angehauchten und eben nicht angelsächsisch und freihändlerisch orientierten Kapitalismus. Letztere Sichtweise gewann vor allem mit der Schaffung der europäischen Währungsunion an Bedeutung, weil hieraus auch eine zunehmende Kohärenz zwischen deutscher und französischer Wirtschaftspolitik und den daraus resultierenden Vorstellungen zur europäischen Integration und insbesondere zur Geld- und Finanzpolitik erwuchs.

Die Unklarheiten der politischen Verwendung des Begriffs lagen zu einem Großteil allerdings schon darin begründet, dass die oft bemühten „Gründerväter“ der Sozialen Marktwirtschaft ganz unterschiedliche Anknüpfungspunkte und Legitimationsmöglichkeiten boten: Wer mittelstandspolitische und föderalistische Überlegungen hegte, konnte den dezentristischen Wilhelm Röpke ins Feld führen, wer auf die Bedeutung von Regelhaftigkeit und Konstanz der Wirtschaftspolitik abzielte, konnte den strengen Ordnungstheoretiker Walter Eucken und andere Repräsentanten der Freiburger Schule anführen, und wem mehr der soziale Ausgleich der wettbewerblichen Ergebnisse und der konjunkturpolitische Aktivismus am Herzen lag, konnte sich auf Alfred Müller-Armack berufen. Entsprechend vielschichtig waren die geistigen Quellen, auf die das Leitbild der „Sozialen Marktwirtschaft“ zurückgeführt und in deren Kontext es ideenhistorisch zu verankern war (z. B. v. Prollius 2006; Hasse 2002).

Was dabei auch in der wissenschaftlichen Diskussion um den Gehalt der Konzeption „Soziale Marktwirtschaft“ regelmäßig zu kurz kam, war der Blick auf die Vorstellungen und Triebkräfte der Protagonisten der Wirtschaftspolitik der frühen Bundesrepublik selbst. Namentlich galt dies für den ersten Bundeskanzler Adenauer, der bislang kaum als Wirtschafts- oder Sozialpolitiker wahrgenommen wird. Aber auch die originären Vorstellungen Ludwig Erhards blieben in den dogmenhistorischen Darstellungen und in den Programmdebatten seltsam blass. Auch diejenigen, die mit Erhards dezidiert freiheitlichen Ordnungsvorstellungen sympathisierten, verstanden ihn oftmals lediglich als Vollstrecker fremder Ideen, nicht aber als eigenen Kopf, der sich beispielsweise in seinen konjunkturpolitischen Vorstellungen durchaus mehr Spielraum herausnahm, als nahestehende Theoretiker wie Walter Eucken oder gar Friedrich August von Hayek zugestanden hätten.

Vor diesem Hintergrund legte die Stiftung Bundeskanzler-Adenauer-Haus in Rhöndorf im Frühjahr 2019 im Rahmen der „Rhöndorfer Ausgabe“ eine über 220 Dokumente umfassende Quellenedition zur Wirtschaftspolitik der frühen Bundesrepublik 
vor (Geppert und Schwarz 2019). In einer Mischedition zeichnete sie in Briefen, Protokollen, Reden, Vermerken und Positionspapieren ein detailliertes Bild über wirtschaftspolitische Entscheidungsprozesse der frühen Bundesrepublik. In der Summe erscheinen diese keineswegs als unerklärliches „Wunder“ (vgl. schon Hunold 1953), geschweige denn als die mühelose Verwirklichung eines theoretischen Ideals, das von anderer Hand entworfen worden wäre. Vielmehr präsentiert die Edition einen durchaus komplexen Aushandlungsprozess mit Inkonsistenzen und Kompromissen, wie sie in der praktischen Politik unvermeidlich sind. Sie wirft auch Schlaglichter auf die konzeptionellen Vorstellungen Adenauers, Erhards und anderer Protagonisten und lässt erkennen, dass nicht nur blanker Pragmatismus herrschte.

Diese Edition war Anlass und Grundlage für eine interdisziplinäre „Rhöndorfer Debatte“, die im November 2019 in Berlin stattfand und Ökonomen, Zeithistoriker, Juristen zusammenbrachte. Dabei ging es zum Einen um den zeithistorischen Kontext der "Sozialen Marktwirtschaft“ in der frühen Bundesrepublik - und dies vor allem mit Blick auf die konzeptionellen Vorstellungen Adenauers und Erhards. Zum Anderen ging es auch um die Frage, inwieweit die Wirtschafts- und Sozialpolitik der Bundesrepublik als Umsetzung dieser konzeptionellen Vorstellungen verstanden werden kann, schließlich auch um die europapolitische und programmatische Gegenwärtigkeit der Konzeption.

In diesem Sonderheft sind einige Impulsvorträge dieser „Rhöndorfer Debatte“ versammelt. Holger Löttel, der Bearbeiter der Edition, zeichnet in einem erweiterten Beitrag das wirtschaftspolitische Denken Konrad Adenauers nach. Richard Reichel richtet in seinem ebenfalls gründlich ausgearbeiteten Beitrag den Blick auf das originäre Denken Ludwig Erhards, der keineswegs als bloßer Umsetzer fremder Ideen, etwa von Alfred Müller-Armack oder Walter Eucken, verstanden werden kann. Zwei pointierte Beiträge von Michael von Prollius und Roland Tichy vermitteln anschaulich den Duktus der Diskussionen: Während von Prollius die Umsetzung der Wirtschafts- und Sozialpolitik der frühen Bundesrepublik kritisch diskutiert, geht Roland Tichy der Frage nach, welche Orientierungspunkte sich aus der frühen Europapolitik Konrad Adenauers und Ludwig Erhards für die aktuellen Kontroversfragen der europäischen Integration gewinnen lassen.

Als die Debatte stattfand, war noch nicht absehbar, dass die Corona-Pandemie die deutsche und europäische Wirtschaftspolitik vor ungeahnte Herausforderungen stellen sollte. Zwei zusätzliche Beiträge greifen daher über die ursprüngliche Veranstaltung hinaus und bilanzieren das Krisenjahr 2020/2021 und die Fernwirkungen der Corona-Pandemie auf die Wirtschafts- und Sozialordnung der Bundesrepublik. Der Berliner Zeithistoriker Paul Nolte unternimmt eine zeit- und ideenhistorische Einordnung der aktuellen Entwicklung und betont dabei das handlungsleitende Bedürfnis nach Stabilisierung. Der Präsident des RWI Leibniz-Instituts für Wirtschaftsforschung, Christoph M. Schmidt, befasst sich aus ökonomischer Warte mit den Fragen nach der richtigen Krisen- und Wachstumsstrategie. 
So heterogen die geistigen Quellen der „Sozialen Marktwirtschaft“ auch waren und so durchwachsen-pragmatisch die Wirtschaftspolitik auch in den 1950er-Jahren schon war, so groß ist doch die Verlockung, danach zu fragen, was Ludwig Erhard als „Corona-Manager“ heute tun würde (Höning 2020) oder ob sich aus dem zeitund ideenhistorischen Kontext der ,Sozialen Marktwirtschaft“ und aus der politischen Praxis der Ära Adenauer/Erhard Orientierung für die Ordnungsprobleme der Gegenwart ableiten lässt. Ein paar Denkanstöße seien an dieser Stelle trotz aller Vorbehalte erlaubt.

\subsection{Konjunkturpolitik}

Am unproblematischsten erscheint das Feld der Konjunkturpolitik. Ludwig Erhard betrieb eine ausgesprochen sensible und aktive Konjunkturpolitik - durchaus bis an hart an die Grenzen dessen, was akademische Vertreter des Ordoliberalismus goutierten - und bisweilen auch darüber hinaus. So hätte auch er in der CoronaPandemie wohl viele Hebel in Bewegung gesetzt, um wirtschaftliche Akteure zu stabilisieren. Instrumente wie das Kurzarbeitergeld und die Mehrwertsteuerabsenkung wären ihm ganz und gar unproblematisch erschienen. Mit Nachdruck hätte er sich wohl für liquiditätsfördernde Krisenhilfen stark gemacht. Vermutlich hätte er dabei aber weniger als im Krisenjahr 2020/2021 auf direkte Zahlungen gesetzt und stattdessen mehr auf die Ausschöpfung der Möglichkeiten für Verlustrückträge im Steuerrecht oder Stundungsmöglichen bei Sozialversicherungsbeiträgen. Er hätte aber stärker im Blick gehabt, dass bloße Stabilisierungsmaßnahmen auch die Gefahr bergen, die Krise zu verlängern und deren Kosten in die Höhe zu treiben. So hätte er mehr Wert darauf gelegt, die Anpassungsfähigkeit der Akteure zu erhöhen, und intensiver auf strukturelle Reformen gedrängt, um dauerhaft die Wettbewerbsfähigkeit der wirtschaftlichen Akteure zu stärken - wohlwissend, dass sich viele Geschäftsmodelle nicht einfach so wieder in Gang setzen lassen, sondern dass es nun umso mehr auf unternehmerischen Erfindungsreichtum und Wagemut ankommt.

\subsection{Dezentrale Innovation}

Für die Konzeption der Sozialen Marktwirtschaft war grundlegend die Etablierung eines freien Preissystems und der Konkurrenz der Anbieter. Vollständig gelangen weder die Freigabe der Preise noch die Durchsetzung des Wettbewerbs. Allein die durchaus umfangreiche Betätigung des Staates selbst stand dem entgegen. Aber die Durchsetzung des Wettbewerbs war doch hinreichend konsequent, um dezentrale, unternehmerisch geprägte Innovationsprozesse in Gang zu setzen, die auf lange Sicht wohlstandsfördernd wirkten. Aktuell haben die Tendenzen zur staatlichen Lenkung von Innovationsprozessen jedoch wieder deutlich zugenommen. Der Glaube an die technologie-, industrie- und regionalpolitische Steuerungsfähigkeit von Förderstrukturen verschiedenster Art ist auffällig und gewinnt auch im Hinblick auf klimapolitische Ziele weitere Attraktivität. Zielführender wäre es allerdings, wenn der Staat sich in dieser Hinsicht eine größere Zurückhaltung auferlegen würde - al- 
so den Grundsatz der Technologieoffenheit in seinen Förderstrukturen beachtet und weniger interventionistische Förderlogiken verfolgen würde. Dann würden viele Akteure aus Wissenschaft und Wirtschaft weniger Energie darauf wenden, politischen Vorgaben für Innovation nachzueifern, und sich stattdessen stärker darauf ausrichten, in freien Suchprozessen dezentrales Wissen zu nutzen und Verbraucherbedürfnisse $\mathrm{zu}$ befriedigen.

\subsection{Wettbewerblicher Klimaschutz}

Umweltpolitik war zu Erhards Zeiten noch kein relevantes Thema. Aber aus der Konzeption des Wettbewerbs ergeben sich auch wichtige Leitlinien für eine nachhaltige Gestaltung der Klimapolitik. In ihr dominieren derzeit in hohem Maße interventionistische Ansätze der Förderung und Besteuerung, die bisweilen zueinander im Widerspruch stehen und Hersteller, Händler und Verbraucher von klimarelevanten Produkten und Dienstleistungen verwirren. Anschluss- und Benutzungszwänge, wie sie insbesondere in der Wärmeversorgung vorgenommen werden, schränken die Wahlfreiheit des Verbrauchers ein, sorgen für lokale Monopole und behindern dezentrale Innovationsprozesse. Es mangelt dagegen nach wie vor an einem konsistenten und transparenten Preissystem, wie es letztlich wohl am besten über einen globalen Emissionshandel herzustellen wäre. Er würde im Sinne von Walter Euckens regulierendem Prinzip der „Wirtschaftsrechnung“ externe Kosten internalisieren und Anreize zur Vermeidung von Verschmutzungseffekten setzen (Eucken 1990, S. 301ff). Es spricht viel dafür, dass ein ausufernder Dirigismus und eine immer undurchsichtiger und hektischer werdende Förderpolitik nicht geeignet sind, ambitionierte Klimaziele zu erreichen, sondern dass es dazu einer funktionierenden Wettbewerbs- und Innovationsordnung bedürfte.

\subsection{Offene Märkte}

Der wirtschaftspolitische Erfolg der frühen Bundesrepublik beruhte auch darauf, dass die Integration in die Weltwirtschaft Schritt für Schritt gelang - mit einem klaren Bekenntnis zum Freihandel und mit einer ausgeprägten Exportorientierung. Der Abbau von Handelshemmnissen und die Konvertibilität der Währung geschahen weder über Nacht noch vollständig. Gleichwohl gehörten sie zum Kernbestand der Sozialen Marktwirtschaft als wirtschaftspolitischer Konzeption. In dieser Hinsicht bestanden grundlegende Unterschiede zur französischen Staatstradition, die immer wieder auf die Karte des Merkantilismus und Protektionismus gesetzt hat. Aktuelle Tendenzen eines sanften Protektionismus auf europäischer Ebene, wie sie im Begriff der ,strategischen Autonomie“ zur Geltung kommen, vertragen sich mit den ursprünglichen Vorstellungen einer „Sozialen Marktwirtschaft“ nicht. Es macht einen grundlegenden Unterschied, ob man sich vor dem Wettbewerb anderer schützen will, oder ob man die eigene Wettbewerbsfähigkeit steigert, indem man sich dem Wettbewerb stellt (s. dazu Kronberger Kreis 2020). 


\subsection{Nachhaltige Staatsfinanzen}

$\mathrm{Zu}$ den Markenzeichen der frühen Bundesrepublik gehörte bis in die Mitte der 1960er-Jahre die Haushaltsdisziplin. Hinter dieser - allmählich aufgeweichten Grundhaltung steckte nicht die buchhalterische Frage, ob und in welchem Maße man sich Verschuldung leisten könne. Dahinter stand die Überzeugung, dass die Staatstätigkeiten nicht, soweit es eben finanzierbar wäre, ausgedehnt werden müssen, sondern dass sie auf vernünftige und begründete Zwecke zu begrenzen seien. Es war die Vorstellung, dass der Staat nicht so sehr durch eigene wirtschaftliche Aktivitäten oder durch stimulierende Eingriffe wirken solle, sondern vor allem als Garant des Wettbewerbs. Der Staat solle mit seinen öffentlichen Investitionen nicht an die Stelle privater Investitionen treten, sondern Voraussetzungen dafür schaffen, dass private Investitionen wieder getätigt würden. Vor diesem Hintergrund hätte Ludwig Erhard vermutlich eine vorübergehend höhere Verschuldung zur Bewältigung der Krise als unvermeidbar angesehen, aber er hätte großen Wert darauf gelegt, dass eine zügige und konsequente Rückführung der Verschuldung betrieben wird.

\subsection{Grenzen der Sozialpolitik}

Die Politik der Sozialen Marktwirtschaft, wie sie Ludwig Erhard verstand, war auf Vermögensbildung, Sparen und Eigenvorsorge angelegt. Nur subsidiär kam für ihn der Staat mit sozialpolitischen Leistungen in Betracht. Selbst Konrad Adenauer, der mit der Rentenreform 1957 einen großen Schritt in die Kollektivierung der Vorsorge beschritt, teilte vor dem Hintergrund seiner sozialethischen Prämissen diesen Grundgedanken der Eigenvorsorge und der Subsidiarität. Heutige Tendenzen, Zinsen künstlich niedrig zu halten, um damit die Kosten der Staatsverschuldung niedrig zu halten, vertragen sich mit diesen Prämissen kaum. Sie schwächen die Möglichkeiten der Eigenvorsorge und fördern ein Staatsverständnis, das auf eine umfassende Alimentierung der Bürger angelegt ist. Erhard wäre heute aus Sorge um eine freiheitlich gesinnte Gesellschaft ein scharfer Kritiker dieser Tendenzen, die Bürger in die Abhängigkeit von staatlicher Alimentierung und Umverteilung zu bringen.

\subsection{Subsidiarität als Integrationsprinzip}

Eine Kontroverse der Gegenwart ist schon in den 1950er-Jahren angelegt: die Frage nach der ordnungspolitischen Qualität der europäischen Integration. Seinerzeit wurde die unterschiedlichen Akzente bei Adenauer und Erhard deutlich: Der erste Bundeskanzler sah die ökonomische Verflechtung als eine Methode, dass die europäischen Nachbarn gemeinsame Interessen definieren: aus der wechselseitigen Abhängigkeit sollte Sicherheit für alle Beteiligten erwachen. Auch Erhard war von der Vorstellung geleitet, dass die ökonomische Integration friedensstiftend wirken kann. Bei ihm war allerdings die Grundidee eines kapitalistischen Friedens greifbarer, denn er plädierte nachdrücklich für den Freihandel als Pendant zur wirtschaftlichen

Freiheit im Inneren. Deshalb stand er auch allen Tendenzen kritisch gegenüber, dass die EWG sich im Agrarmarkt oder in der Industriepolitik als wirtschaftlicher Block 
nach außen abschottete. Daraus resultierten nicht zuletzt unterschiedliche Leitideen zur europäischen Integration.

Die Schlussfolgerungen daraus für die Gegenwart liegen auf der Hand: Die politische Logik der europäischen Einigung trägt letzten Endes nur so weit, wie die Integration auch in wirtschaftlicher Hinsicht förderlich ist. Die Kollektivierung von Staatsschulden, die Zentralisierung von Interessenkonflikten durch eine ausgreifende Konjunktur- oder Sozialpolitik, die Verschleierung von politischer Verantwortung im Mehrebenensystem, ein einseitiger Trend zur Vergemeinschaftung von immer mehr Politikfeldern, industriepolitischer Steuerungseifer und Abschottungstendenzen nach außen überfordern und gefährden die Integration auf lange Sicht. Die Rückbesinnung auf den Grundsatz der Subsidiarität, eine stabilitätsorientierte Geldpolitik, die Betonung von Wettbewerbsfähigkeit und einer offenen Innovationskultur, die Klärung von Verantwortlichkeiten zwischen den Ebenen und Institutionen und die Stärkung demokratischer Kontrollmöglichkeiten wären statt dessen geboten, wenn man auf den politischen und wirtschaftlichen Erfolg der Integration aus ist und die Fliehkräfte eindämmen will.

Funding Open Access funding enabled and organized by Projekt DEAL.

Open Access Dieser Artikel wird unter der Creative Commons Namensnennung 4.0 International Lizenz veröffentlicht, welche die Nutzung, Vervielfältigung, Bearbeitung, Verbreitung und Wiedergabe in jeglichem Medium und Format erlaubt, sofern Sie den/die ursprünglichen Autor(en) und die Quelle ordnungsgemäß nennen, einen Link zur Creative Commons Lizenz beifügen und angeben, ob Änderungen vorgenommen wurden.

Die in diesem Artikel enthaltenen Bilder und sonstiges Drittmaterial unterliegen ebenfalls der genannten Creative Commons Lizenz, sofern sich aus der Abbildungslegende nichts anderes ergibt. Sofern das betreffende Material nicht unter der genannten Creative Commons Lizenz steht und die betreffende Handlung nicht nach gesetzlichen Vorschriften erlaubt ist, ist für die oben aufgeführten Weiterverwendungen des Materials die Einwilligung des jeweiligen Rechteinhabers einzuholen.

Weitere Details zur Lizenz entnehmen Sie bitte der Lizenzinformation auf http://creativecommons.org/ licenses/by/4.0/deed.de.

\section{Literatur}

Bundesministerium für Wirtschaft und Energie (2019). Nationale Industriestrategie 2030. Strategische Leitlinien für eine deutsche und europäische Industriepolitik. Berlin: Bundesministerium für Wirtschaft und Energie. https://www.bmwi.de/Redaktion/DE/Publikationen/Industrie/nationaleindustriestrategie-2030.html

Eucken, W. (1990). Grundsätze der Wirtschaftspolitik. Tübingen: Mohr Siebeck.

Geppert, D., \& Schwarz, H.-P. (Hrsg.). (2019). Adenauer. Rhöndorfer Ausgabe. Konrad Adenauer, Ludwig Erhard und die Soziale Marktwirtschaft. Paderborn: Schöningh. Bearb. von Holger Löttel

Habeck, R. (2019). Deutschland denkt zu klein, das ist zu anspruchslos. In Die Welt. https://www.welt.de/ 203480482. Zugegriffen: 30. April 2021.

Hasse, R. (Hrsg.). (2002). Lexikon Soziale Marktwirtschaft. Wirtschaftspolitik von A bis Z. Paderborn: Schöningh.

Hayek, F. A. (1976). The mirage of social justice. Law, legislation and liberty, Bd. 2. Chicago: University of Chicago Press.

Hombach, B. (1998). Aufbruch. Die Politik der neuen Mitte. Düsseldorf, München: Econ.

Höning, A. (2020). Ludwig Erhard, der Corona-Manager. In Rheinische Post, 7. Okt. 2020.

Hunold, A. (1953). Wirtschaft ohne Wunder. Aufsätze. Erlenbach, Zürich: Rentsch. 
Kronberger Kreis (2020). Kein Rückzug in die Festung Europa! Berlin: Stiftung Marktwirtschaft. Merz, F. (2010). Mehr Kapitalismus wagen. Wege zu einer gerechteren Gesellschaft. München: Piper.

Oakeshott, M. (2000). Zuversicht und Skepsis. zwei Prinzipien neuzeitlicher Politik. Berlin: Alexander Fest.

v. Prollius, M. (2006). Deutsche Wirtschaftsgeschichte nach 1945. Göttingen: Vandenhoeck \& Ruprecht. Rüttgers, J. (2007). Die Marktwirtschaft muss sozial bleiben. Köln: Kiepenheuer.

Schiller, K. (1964). Der Ökonom und die Gesellschaft. Das freiheitliche und das soziale Element in der modernen Wirtschaftspolitik. Stuttgart: G. Fischer.

Wagenknecht, S. (2011). Freiheit statt Kapitalismus. Frankfurt a.M.: Eichborn. 